\title{
A comparative study of topical sucralfate versus honey dressing in the management of diabetic foot ulcer
}

\author{
Reddy M.V. ${ }^{1}$, Inamdar P. ${ }^{2}$, Gogineni M. ${ }^{3}$ \\ ${ }^{1}$ Dr. M. Venkata Reddy, Professor, ${ }^{2}$ Dr. Padmanabh Inamdar, Associate Professor, ${ }^{3}$ Dr. Gogineni Mahesh, Senior \\ Resident, all authors are affiliated with Department of Surgery, Mamata Medical College and Hospital, Khammam, \\ Telangana, India.
}

Corresponding Author: Dr. Padmanabh Inamdar, Associate Professor, Department of Surgery, Mamata Medical College and Hospital, Khammam, Telangana, India. E-mail: docpadmanabh@gmail.com

\begin{abstract}
Background and Objectives: Local wound care is one of the mainstays in the management protocol for diabetic foot ulcers. Sucralfate and honey are inexpensive and easily available agents for local dressing. They are highly efficacious in achieving faster and better healing. Our study compares the effect of topical sucralfate with honey dressings in the management of diabetic foot ulcers. Methods: We conducted a prospective study on 50 patients of Diabetic foot ulcer admitted indoor and were evaluated clinically. Patients were divided into Group A ( $\mathrm{n}=25)$ and Group B ( $\mathrm{n}=25)$. Group A patients treated with topical application of Sucralfate and Group B patients were treated with topical honey. Wounds in both groups were inspected at the end of 7 days, 14 days and 21 days. Results in the two groups were compared and interpreted. Results: In our study, mean ulcer size was reduced in both the groups on day 7, day 14, and day21 ( $\mathrm{p}>0.5$ ). The percentage of reduction in mean ulcer size in Group A was 59.53\% compared to 55.05\% in Group B at the end of 21 days ( $\mathrm{p}$ value $>0.05)$. Completely epithelized ulcers were $10(40 \%)$ in Group A and $9(36 \%)$ in Group B respectively. Complete epithelization of ulcer or ulcer bed ready for split skin grafting (primary end point of study) was 21.04 days in Group A and 23.20 days in Group B (P value < 0.05). Conclusion: Even though topical sucralfate and honey were both found to be effective in enhancing the wound healing process in diabetic foot ulcers, topical sucralfate was found to be better when compared to honey for local application.
\end{abstract}

Keywords: Diabetes mellitus, Diabetic Foot ulcer, Topical honey, Topical sucralfate

\section{Introduction}

Diabetes mellitus is characterized by chronic hyperglycaemia with disturbances of carbohydrate, fat, and protein metabolism resulting from defects in insulin secretion, insulin action or both. It is the leading cause of end stage renal disease, a major cause of non traumatic amputations, responsible for $30 \%$ of the preventable blindness and a leading cause of cardiovascular mortality. Diabetes mellitus (DM) is one of the main problems in health systems and a global public health threat that has increased dramatically over the past 2 decades. According to epidemiological studies, the number of patients with DM increased from about 30 million cases in 1985, 177 million in 2000, 285 million in 2010, and estimated if the situation continues, more than 360 million people by 2030 will have DM [1].

Manuscript Received: $28^{\text {th }}$ August 2019

Reviewed: $7^{\text {th }}$ September 2019

Author Corrected: $14^{\text {th }}$ September 2019

Accepted for Publication: $17^{\text {th }}$ September 2019
Diabetic foot is one of the major complications of diabetes mellitus. One in every six people with diabetes will have a foot ulcer during his /her lifetime [2]. In total, it is estimated that $15 \%$ of patients with diabetes will suffer from DFU during their lifetime [3]. Diabetic foot ulcers are extremely debilitating and difficult to treat. Multidisciplinary management, patient education, glucose control, debridement, offloading, infection control, and adequate perfusions are the mainstays of standard care endorsed by most practice guidelines.

Local wound care is one of the mainstays in the management protocol for diabetic foot ulcers. Currently there is a wound care revolution and a wide variety of dressings are available, yet there is no ideal dressing. During the last two decades a wide variety of innovative dressings have been introduced in wound healing, such as Benzoyl peroxide, collagen, insulin, oxygen therapy, sucralfate, honey and vinegar. Among people from a 


\section{Original Research Article}

lower socioeconomic background, cost of treatment plays an important role in compliance and seeing the treatment to completion. As both sucralfate and honey are inexpensive and easily available agents, we undertook a study to know their efficacy in promoting wound healing in diabetic foot ulcers. As there is a need to optimize the ideal type of dressing in diabetic foot ulcers, we undertook the study with an aim to compare effect of topical sucralfate with topical honey dressings in the management of diabetic foot ulcers.

\section{Patients and Methods}

Study design- This was prospective observational study to compare topical sucralfate versus honey dressing in the management of diabetic foot ulcer.

Study sample - Study was conducted in Department of General surgery from October 2016 to September 2018. Study sample consisted of 50 patients of diabetic foot ulcer in Mamata General Hospital. Institutional ethical committee (IEC) approval and patient consent was taken before beginning the study.

\section{Inclusion criteria}

- Patients between 25 to 75 years of age with type 2 diabetes mellitus with diabetic foot ulcers of Wagner's grade 1 and 2

- Size of ulcer less than $10 \times 10 \mathrm{cms}$

- Patients willing to take part in the study.

\section{Exclusion criteria}

- Patients with chronic venous insufficiency of lower limbs with dermal changes and lymphedema

- Patients with uncontrolled diabetes with severe comorbid medical conditions

- Immunocompromised patients

- Associated osteomyelitis, Skin malignancy

- Patients unwilling for the study.

Statistical method- Unpaired $t$ test was used for analysis of continuous data. Pearson's $x^{2}$ test was used for analysis of categorical data. Differences were considered statistically significant, if $\mathrm{P}<0.05$. IBM SPSS Statistics for Windows, version 24 (IBM Corp.,
Armonk, N.Y., USA) software program was used for statistical calculations. Analytical data obtained was compared and discussed with the data available in the literature.

Data collection- Patients were divided into 2 groups for study purpose. Group A $(n=25)$ patients were treated with sucralfate dressing, whereas Group B $(n=25)$ patients were treated with honey.

After taking an informed and written consent of patients having diabetic ulcer, thorough history, clinical examination was carried out. Relevant investigations like Hemoglobin ( $\mathrm{Hb}$ ), Total Leukocyte count (TLC) Fasting blood sugar (FBS), Post prandial blood sugar (PPBS), and Glycosalated Hemoglobin (HbA1c) were done in both the groups.

Before application, culture \& sensitivity swab of all ulcers were taken and cleaned with normal saline. Debridement of dirty and crusted wound was done. Good glycemic control was achieved using oral hypoglycemic and/or Insulin therapy. All patients were treated with appropriate antibiotics after culture and sensitivity report. Off-loading of pressure from the affected area was used in both the groups.

Commercially available sucralfate suspension (Sucrafil) was taken in a small sterile bowl, ulcer surface was coated with sucralfate suspension. Ulcer was initially cleaned with normal saline and sterile gauze sucralfate suspension was placed over the wound. Honey dressing was applied in the same manner as sucralfate with commercially available sterilized honey. At the end of 7 days, 14 days and 21 days the wounds in both groups were inspected. Data was collected in proforma and entered into excel sheet for comparison. The end point of study was complete wound epithelializat ion or appearance of granulation tissue, which ultimately lead to spilt skin grafting or secondary healing; whichever is earlier. Rate of wound healing was calculated as the difference between the primary wound on the day 1 and at end of 7days, 14 days and 21 days. Ulcer measurements were taken using ruler (in $\mathrm{cm}$ ) as two largest perpendicular diameters. The ulcer area was measured in $\mathrm{cm}^{2}$ by multiplying these two diameters.

\section{Results}

The Mean age of subjects in the present study in Group A was 57.28 years and in group B was 57.04 years. The mean ulcer size at Day 1, Day 7, day 14 and day 21 is shown in Fig 1. Size of ulcer was comparable though statistically not significant. In the present study, the percentage of reduction in mean ulcer size in Group A was 59.53\% compared to $55.005 \%$ in Group B at the end of 21 days ( $p$ value $>0.05$ ). Average number of dressing changed in Group A was 19.80 whereas in Group B it was 21.88 with $\mathrm{P}$ value $<0.05$ which was statistically significant. 


\section{Original Research Article}

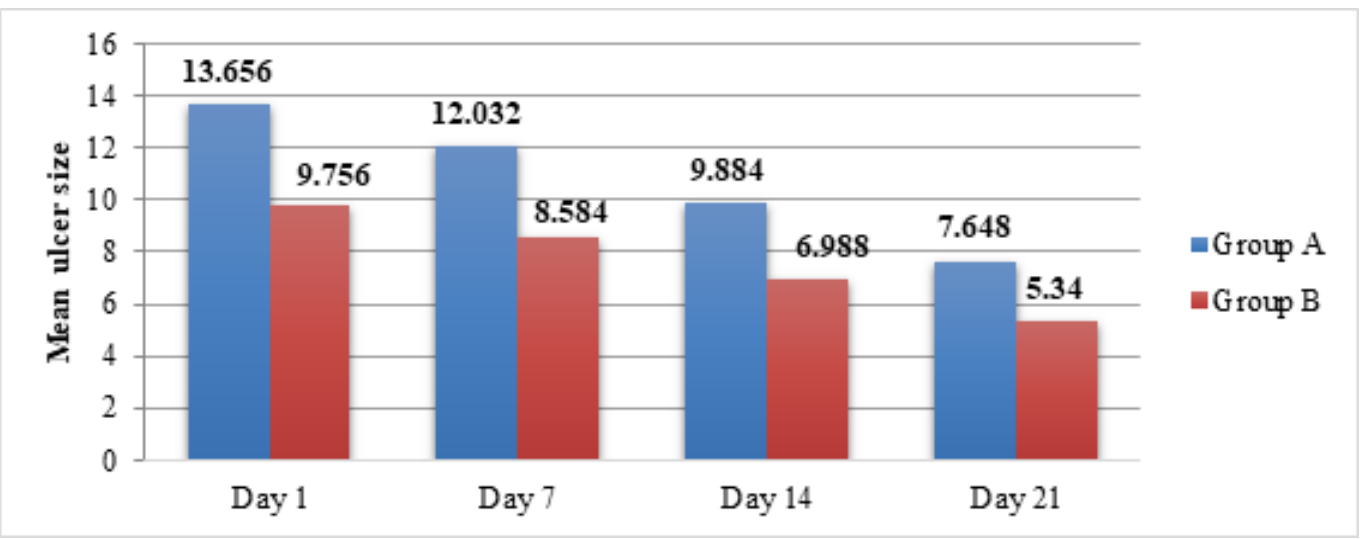

Fig.1: Mean ulcer size at day 1, day 7, day 14 and day 21

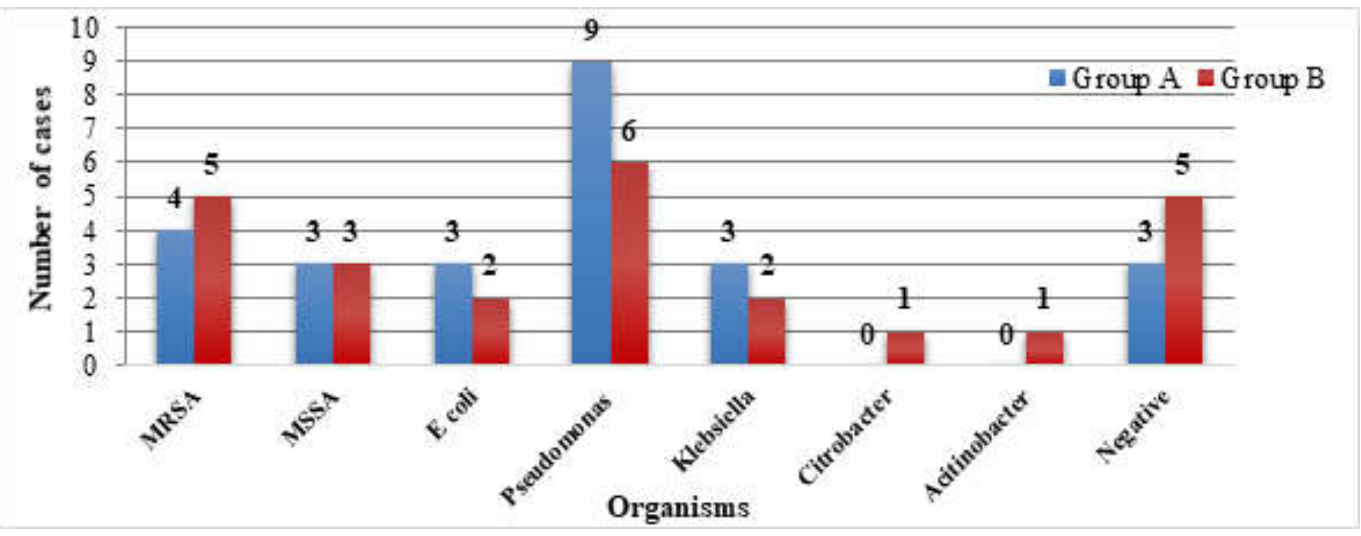

Fig-02: Organisms isolated during the study

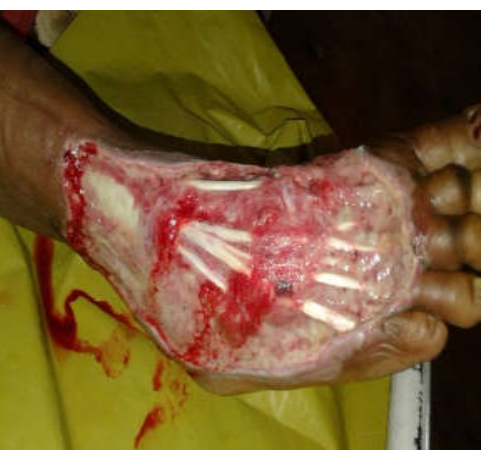

Before

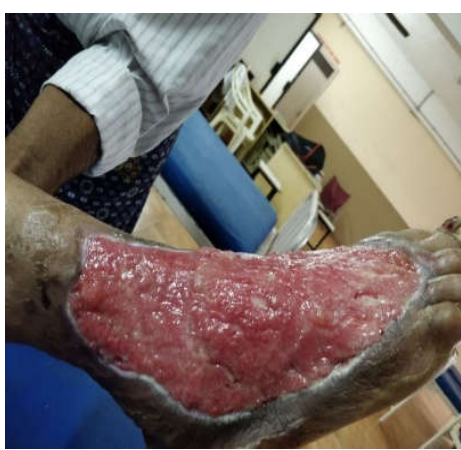

After

Fig.-03: Wound before and after treated with topical sucralfate dressing

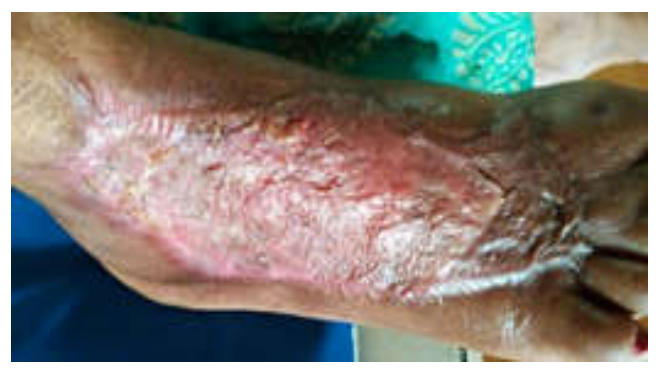

Fig.-04: Wound after split skin grafting in sucralfate group 


\section{Original Research Article}

Number of patients with completely epithelized ulcers were 10 (40\%) in Group A and 9 (36\%) in Group B respectively. Skin grafting was done in $15(60 \%)$ and $16(64 \%)$ cases in Group A and Group B respectively. Bacteriological profile of ulcer is shown in Fig 2. The bacterium that was cultured most in the ulcers was Pseudomonas aeruginosa followed by MRSA.

Table-1: Table showing overview of study patients $(n=50)$.

\begin{tabular}{|l|c|c|c|}
\hline & Group A (n=25) & Group B (n=25) & p value \\
\hline Age (Years) & 57.28 & 57.04 & \\
\hline Sex & & & \\
\hline Male & 16 & 17 & \\
\hline Female & 9 & 8 & \\
\hline Percentage reduction in mean ulcer size & $59.53 \%$ & $55.01 \%$ & $>0.05$ \\
\hline Average no. of ulcer dressing changed & 19.8 & 21.88 & $<0.05$ \\
\hline Completely epithelialized ulcers & $10(40 \%)$ & $9(36 \%)$ & $>0.05$ \\
\hline Split skin grafting & $15(60 \%)$ & $16(64 \%)$ & $>0.05$ \\
\hline Mean duration for ulcer heal or SSG & 21.04 days & 23.20 days & $<0.05$ \\
\hline Mean Ulcer size (cm2) & & & \\
\hline Day 1 & 13.656 & 9.756 & $>0.05$ \\
\hline Day 7 & 12.032 & 8.584 & $>0.05$ \\
\hline Day 14 & 9.884 & 6.988 & $>0.05$ \\
\hline Day 21 & 7.648 & 5.34 & $>0.05$ \\
\hline
\end{tabular}

The mean duration for complete epithelization of ulcer or ulcer bed ready for split skin grafting which is primary end point of study was 21.04 days in Group A and 23.20 days in Group B which was statistically significant $(\mathrm{P}$ value $<0.05)$. Relevant findings in study patients are summarized in Table 1.

\section{Discussion}

An ideal dressing is every surgeon's desire, a dressing that promotes chronic ulcer healing without any complications. Successful wound dressing should keep the wound moist and be devoid of any adverse reactions such as infection, maceration and allergy. Diabetic ulcers are chronic wounds, stuck in inflammation phase and shows cessation of epidermal growth. Wound dressings have evolved from the status of providing physical protection to the raw surface, absorbing exudates and controlling local infections by local medications to the level of providing an adequate environment promoting wound healing. This has been achieved by modern wound dressing agents, which promote granulation tissue formation.

Sucralfate is a commonly used antacid. It is a basic aluminum salt of sucrose octasulfate. It acts as a mechanical barrier because of a strong electrostatic interaction with proteins at the ulcer site [4]. Sucralfate has also been shown to have antibacterial activity [5]. Recent studies have also shown that it is structurally similar to heparin, and hence has angiogenic properties [6].
In present study there was similar age and sex distribution. Mean ulcer size when compared on day 1, day 7 , day 14 and day 21 were comparable in both the groups and showed decreasing in mean size similar to study done by Akarsh, Y. G. et al [7].

The percentage of reduction in mean ulcer size in the sucralfate group was $59.53 \%$ at the end of 21 days which was similar to study done by Nagalakshmi G et al [8], Dr. Akarsh, Y. G. et al [7]. At the end of study period complete epithelialization of ulcer was achieved in $10(40 \%)$ subjects in sucralfate group and $9(36 \%)$ subjects in honey group which is congruent with study done by Eldeen $\mathrm{M}$ et al [9], Edmonds $\mathrm{M}$ et al [10], Imran $\mathrm{M}$ et al [11].

However, in the present study complete ulcer epithelialization rate is comparatively less when compared to other studies. This may be because; the evaluation period and mean ulcer size were different between these studies. The study period in Edmonds $M$ et al [10] study was 20 weeks whereas the study period in study done by Imran M et al [11] was 120 days. 


\section{Original Research Article}

The number of cases who underwent split skin grafting in the present study were less when compared to the other studies like Prabhakar S et al [12], Shukrimi A et al [13]. This may be because; the endpoint of the present study is different from other studies mentioned above. Mean duration of complete epithelization was significantly shorter in sucralfate group results which, correlate well with study done by Nagalakshmi G et al [08], Agarwal S et al [14], Moghazy AM et al [15], Shukrimi A et al [13].

Sucralfate has been shown to accelerate epithelial wound healing by increasing the bioavailability of growth factors, especially FGF, which in turn has been demonstrated to have a pivotal role in angiogenesis, a main phase in epithelial wound healing. In addition, the induction of prostaglandins production as well as cell apoptosis protection by sucralfate can favor the re epithelialization in the wound healing process.

The local delivery of growth factors has been proposed as a tool to improve epithelial wound healing. It will be appropriate to design clinical trials in patients with various types of epithelial wounds to investigate the use of simultaneous topical delivery of sucralfate and one or more growth factors. The encouraging results seen in our study are in concordance with study done by Tsakayannis D et al [16], Tumino G et al [17], who used topical sucralfate to treat diabetic foot ulcers.

In the present study, the bacterium that was cultured most in the ulcers was Pseudomonas aeruginosa followed by MRSA. Pseudomonas aeruginosa was found in $9(36 \%)$ cases in sucralfate group and $6(24 \%)$ cases in honey group. MRSA was found in $4(16 \%)$ subjects of sucralfate group and $5(20 \%)$ subjects of honey group. Findings of our study are comparable to study done by Nagalakshmi G et al [8], Agarwal S et al [14], Moghazy AM et al [15], West et al [18].

Honey has been used in clinical practice for many types of disease for centuries [7]. It is still being used as a dressing material for burn wounds, decubitus ulcers, gunshot wounds and wound dehiscence. It enhances auto debridement by absorbing edematous fluid around the ulcer margins and promotes granulation tissue formation and epithelization [19].

High osmolarity has been considered a valuable tool in the treatment of infections because it prevents the growth of bacteria and encourages healing. This can be achieved by the topical use of honey, which contains up to $40 \%$ fructose, $30 \%$ glucose, $5 \%$ sucrose and $20 \%$ water [20]. Our study has also shown positive results with topical honey dressing similar to study published by Vardi et al [21], Subrahmanyam M [22].

There has been a significance advance in diabetic foot ulcer care in the form of development of wound dressing systems, which stimulate wound healing process by improved granulation tissue formation. There is also development of growth factors which cause molecular manipulation in the wound microenvironment; topical application of these growth factors appears to signal a significant role for their therapeutic use in the treatment of DFU.

Nevertheless, only a single medication growth factor supplementation (PDGF) was approved by the FDA for topical application that has modest success. There is trend towards development of bioengineered skin substitutes which have a major role in chronic wounds. Extensive research is going on in the development of artificial skin substitutes by combining cultured keratinocytes with artificially formed dermal analogues, namely Integra, Allo Derm, polygalactin mesh, human allogenic dermis etc., which has immense potential. It is only a matter of time before a successful approach to the management of chronic wounds is devised.

There are now different types of dressings that can be used to treat diabetic foot ulcers such as absorptive fillers, hydrogel dressings, and hydrocolloids. Advanced therapies for management of diabetic foot ulcer includes hyperbaric oxygen therapy, electrical stimulation, negative pressure wound therapy and growth factors. In selecting dressings for chronic non healing wounds it is recommended that the cost of the product should be considered.

We acknowledge the limitations of present study. Sample size was small, and study was conducted in single institute, various factors other than cost of dressings were not analyzed and it was not a blinded study. We did not compare with other available topical application methods for healing of diabetic foot ulcer. Multicenter trial with large number of patients is needed to establish ideal agent for topical application.

To conclude topical sucralfate and honey were both found to be effective in enhancing the wound healing process in diabetic foot ulcers, but topical sucralfate was found to be slightly better when compared to honey in the enhancement of wound healing in terms of mean duration for complete epithelialization of ulcer or ulcer bed ready for split skin grafting and number of 


\section{Original Research Article}

dressings required. However, further studies with larger population will be needed in the future before topical sucralfate dressing can be added to the wide spectrum of treatment modalities available in the management of diabetic ulcers and ulcers of other etiology.

\section{What the study adds to the existing knowledge?}

Even though several topical solutions are available for local application many of them are costly and beyond reach of many patients. It is a unique study as literature is sparse comparing topical sucralfate with topical honey in diabetic foot patients.Our study shows cheaper alternatives like topical sucralfate and honey can be used in diabetic foot ulcer patients there by reducing economic burden on patients with good efficacy.

\section{Author's contribution}

Dr. M Venkat Reddy: Study design, data analysis and manuscript preparation.

Dr. P Inamdar: Study design, data analysis and manuscript editing and proof reading and manuscript submission.

Dr. G Mahesh: Conceptualization of study, data collection and Manuscript preparation

Conflict of interest: None declared. Funding: Nil, Permission from IRB: Yes

Ethical approval: Taken

\section{References}

1. Yazdanpanah L, Nasiri M, Adarvishi S. Literature review on the management of diabetic foot ulcer. World J Diabetes. 2015;6(1):37-53. doi: 10.4239/wjd.v6.i1.37.

2. Bakker K, van Houtum WH, Riley PC. 2005: The International Diabetes Federation focuses on the diabetic foot. Curr Diab Rep. 2005;5(6):436-440.

3. Leone S, Pascale R, Vitale M, Esposito S. [Epidemiology of diabetic foot]. Infez Med. 2012;20(1): $8-13$.

4. Frykberg RG, Zgonis T, Armstrong DG, Driver VR, Giurini JM, Kravitz SR, et al. Diabetic foot disorders. A clinical practice guideline (2006 revision). J Foot Ankle Surg. 2006; 45(5): S1-66. doi:10.1016/S1067-2516(07) 60001-5

5. Danesh BJ, Duncan A, Russell RI. Is an acid $\mathrm{pH}$ medium required for the protective effect of sucralfate against mucosal injury? Am J Med. 1987;83(3B):11-13. doi:10.1016/0002-9343(87)90820-5
6. Tryba M, Mantey-Stiers F. Antibacterial activity of sucralfate in human gastric juice. Am J Med. 1987; 83(3B): 125-127. doi: 10. 1016/ 0002-9343 (87) 908 41-2

7. Akarsh Y G, Nischal K. A comparative study of topical human placental extract with topical sucralfate in the management of diabetic foot ulcers. Int $\mathrm{J}$ Curr Res. 2016;8(03):28501-28503.

8. Nagalakshmi G, Amalan AJ, Anandan H. Clinical Study of Comparision Between Efficacy of Topical Sucralfate and Conventional Dressing in the Management of Diabetic Ulcer. Int J Scienti Study. 2017; 5 (3): 236-238. doi: 10. 17354/ ijss/ 2017/ 302

9. Eldeen M, Fathey R, Hasaballah A, Basal A. Topical honey versus alginate as dressing for management of wagner's Type 2 diabetic foot ulcer. J Am Sci. 2012; 8(9): 459-464.

10. Edmonds M, Lázaro-Martínez JL, Alfayate-García JM, Martini J, Petit JM, Rayman G, et al. Sucrose octasulfate dressing versus control dressing in patients with neuroischaemic diabetic foot ulcers (Explorer): an international, multicentre, double-blind, randomised, controlled trial. Lancet Diabetes Endocrinol. 2018; 6(3): 186-196. doi: 10.1016/S2213-8587(17)30438-2. Epub 2017 Dec 20.

11. Imran M, Hussain MB, Baig M. A Randomized, Controlled Clinical Trial of Honey-Impregnated Dressing for Treating Diabetic Foot Ulcer. J Coll Physicians Surg Pak. 2015; 25(10): 721-725. doi: 10. 2015 / JCPSP.721725.

12.Prabhakar S, Mathias N, Moosabba MS. Comparative study between phenytoin and sucralfate dressing in diabetic foot ulcers. Med Int J Surg. 2017; 3 (1): 1-3. doi: https:// doi. org/10. 26611/ 1063 111

13. Shukrimi A, Sulaiman AR, Halim AY, Azril A. A comparative study between honey and povidone iodine as dressing solution for Wagner type II diabetic foot ulcers. Med J Malaysia. 2008;63(1):44-46.

14.Agarwal S, Bhardwaj V, Singh A, Khan MH, Goel S, Bharat M, Krishna J. A control clinical trial of honey-impregnated and povidone iodine dressings in the treatment of diabetic foot ulcers among northern Indian subjects. Int Wound J. 2015;6(2):7-10. 


\section{Original Research Article}

15. Moghazy AM, Shams ME, Adly OA, Abbas AH, El-Badawy MA, Elsakka DM, et al. The clinical and cost effectiveness of bee honey dressing in the treatment of diabetic foot ulcers. Diabetes Res Clin Pract. 2010 Sep; 89(3):276-81. doi: 10.1016/j.diabres. 2010. 05.021. Epub 2010 Jun 19.

16.Tsakayannis D, Li WW, Razvi S, Spirito N. Sucralfate and chronic venous stasis ulcers. Lancet. 1994; 343(8894):424-425. doi:10.1016/s01406736 (94)91263-7.

17. Tumino G, Masuelli L, Bei R, Simonelli L, Santoro A, Francipane S. Topical treatment of chronic venous ulcers with sucralfate: a placebo controlled randomized study. Int J Mol Med. 2008;22(1):17-23. doi: https:// doi.org/ 10.3892/ijmm.22.1.17

18. West AP, Abdul S, Sherratt MJ, Inglis TJ. Antibacterial activity of sucralfate against Escherichia coli, Staphylococcus aureus and Pseudomonas aeruginosa in batch and continuous culture. Eur J Clin Microbiol Infect Dis. 1993;12(11): 869-871. doi:10. 1007/ bf02000412

19. Dubhashi SP, Sindwani RD. A comparative study of honey and phenytoin dressings for chronic wounds. Ind J Surg. 2015;77(3):1209-1213. doi: 10.1007/s12262015-1251-6

20. Townsend CM, Beauchamp RD, Evers BM, Mattox KL. $20^{\text {th }}$ Ed. Townsend: Sabiston textbook of surgery.

21. Vardi A, Barzilay Z, Linder N, Cohen HA, Paret G, Barzilai A. Local application of honey for treatment of neonatal postoperative wound infection. Acta Paediatrica. 1998; 87(4): 429-432. doi: https://doi.org/ 10. 1111/j. 1651-2227.1998.tb01473.x

22. Subrahmanyam M. Topical application of honey in treatment of burns. Br J Surg. 1991;78(4):497-498. doi: 10.1002/bjs.1800780435

\section{How to cite this article?}

Reddy M.V, Inamdar P, Gogineni M. A comparative study of topical sucralfate versus honey dressing in the management of diabetic foot ulcer.Surgical Update: Int J surg Orthopedics. 2019;5(4):246-252.doi:10.17511/ijoso.2019.i04.03 